\title{
Design of the Hole-Injection/Hole-Transport Interfaces for Stable Quantum-Dot Light-Emitting Diodes
}

Yuxun Ye ${ }^{1}$, Xuerong Zheng ${ }^{2}$, Desui Chen ${ }^{2}$, Yunzhou Deng' ${ }^{2}$ Dong Chen', Yanlei Hao ${ }^{2}$, Xingliang Dai ${ }^{2 *}$ Yizheng Jin $^{2 *}$

${ }^{1}$ Centre for Chemistry of High-Performance \& Novel Materials, State Key Laboratory of Silicon Materials, School of Materials Science and Engineering, Zhejiang University, Hangzhou, China.

${ }^{2}$ Centre for Chemistry of High-Performance \& Novel Materials, State Key Laboratory of Silicon Materials, Department of Chemistry, Zhejiang University, Hangzhou, China.

Correspondence authors:

Dr. Xingliang Dai (shanfeng@zju.edu.cn), Prof. Yizheng Jin (yizhengjin@zju.edu.cn) 


\section{Experimental Methods}

Materials. Red CdSe/CdZnSe/CdZnS core/shell/shell QDs was purchased from Najing Technology Co. Ltd. TFB (average molecular weight: $~ 50,000 \mathrm{~g} \mathrm{~mol}^{-1}$ ) was purchased from American Dye Source. ZnO nanoparticles were synthesized according to a method reported in the literature ${ }^{1,2}$. Extra dry solvents, chlorobenzene (99.8\%), octane (>99\%), and ethanol (99.5\%) were purchased from Acros.

Device fabrication. PEDOT:PSS solutions (Baytron PVP Al 4083, filtered through a $0.45 \mu \mathrm{m}$ N66 filter) were spin-coated onto the cleaned ITO substrates at 3000 r.p.m. for 60 s, followed by thermal annealing at $150^{\circ} \mathrm{C}$ for $30 \mathrm{~min}$ in air. The PEDOT:PSS-coated substrates were transferred into a nitrogen-filled glove box $\left(\mathrm{O}_{2}<1\right.$ ppm, $\mathrm{H}_{2} \mathrm{O}<1$ ppm). TFB (in chlorobenzene, $\left.12 \mathrm{mg} \mathrm{mL}^{-1}\right)$, QDs (in octane, $\left.15 \mathrm{mg} \mathrm{mL}^{-1}\right)$, small-size $(\sim 2 \mathrm{~nm}) \mathrm{ZnO}$ nanoparticles (in ethanol, $\left.7 \mathrm{mg} \mathrm{mL} \mathrm{m}^{-1}\right)$ and large-size $(\sim 6 \mathrm{~nm}) \mathrm{ZnO}$ nanoparticles (in ethanol, $23 \mathrm{mg} \mathrm{mL}^{-1}$ ) were layer-by-layer deposited by spin coating at 2,000 r.p.m. for 45 s. The TFB layer and the small-size $\mathrm{ZnO}$ layer were thermal annealed at $150^{\circ} \mathrm{C}$ for $30 \mathrm{~min}$ and $80^{\circ} \mathrm{C}$ for $30 \mathrm{~min}$, respectively, before deposition of the next layer. Finally, the samples were transferred into a thermal evaporation system (Trovato 300C) for deposition of the silver cathode $(100 \mathrm{~nm})$, patterned by a shadow mask to provide an active device area of $4 \mathrm{~mm}^{2}$.

For the fabrication of the hole-only devices, $\mathrm{MoO}_{\mathrm{x}} / \mathrm{Au}$ cathode was used to replace the $\mathrm{Ag}$ cathode. For the fabrication of the electron-only devices, the deposition sequence of the layers was altered to $\mathrm{ZnO}, \mathrm{TFB}, \mathrm{ZnO}$ and $\mathrm{Al}$. All devices were encapsulated by covering glass slides with acid-free ultraviolet-curable resin (LOCTITE 3335). 
Regarding the transformation of the bipolar devices to the hole-only devices, the top silver electrodes were delaminated by adhesive tapes. Then, the ZnO-nanoparticle ETLs were etched by a dilute ethanol solution of acetic acid ( $0.5 \%$ volume ratio), followed by washing with pure ethanol for three times. The samples were baked at $60{ }^{\circ} \mathrm{C}$ for $30 \mathrm{~min}$ to remove the residue ethanol, then transferred into a thermal evaporation system for the deposition of the $\mathrm{MoO}_{\mathrm{X}} / \mathrm{Au}$ electrodes.

Oxygen plasma treatment of PEDOT:PSS. The PEDOT:PSS-coated substrates were treated with oxygen plasma before transfer into the glovebox. The samples were loaded into a plasma cleaner (Harrick Plasma, PDC-32G-2) with a controlled oxygen flux of $\sim 50 \mathrm{~mL} \mathrm{~min}{ }^{-1}$ and treated for 4 minutes under oxygen plasma using a maximum power of $\sim 18 \mathrm{~W}$.

Characterizations. The J-V-L characteristics of QLEDs were measured by a home-built QLED-testing system consisting of a Keithley 2400 source meter and an integration sphere (FOIS-1) coupled with a QE-Pro spectrometer (Ocean Optics). The devices were swept from zero bias to forward bias with a scanning rate of $0.1 \mathrm{~V} \mathrm{~s}^{-1}$ and an integration time of 100 milliseconds for each voltage. The EL spectra of devices were synchronously recorded.

The operational lifetimes of QLEDs were measured under ambient conditions (room temperature: $22-24{ }^{\circ} \mathrm{C}$ and relative humidity: $40-60 \%$ ) by using a commercialized system designed by Guangzhou New Vision Opto-Electronic Technology Co., Ltd. All devices were bias-stressed for at least 5 hours at a constant current of $50.0 \mathrm{~mA} \mathrm{~cm}^{-2}$ before the test.

The PL spectra of devices and TFB films were recorded by a QE-Pro spectrometer. The devices were excited by a $405 \mathrm{~nm}$ laser with a power density of $\sim 2 \mathrm{~mW} \mathrm{~cm}{ }^{-2}$. The 
measurement setup for the collection of PL spectra was fixed throughout the electrical aging of devices or the optical excitation of TFB film.

The electro-absorption spectra were collected in the reflection mode. A system consisting of a Xenon lamp coupled with a monochromator (Horiba), a waveform generator electrical power (Siglent SDG1032X), a silicon photodiode (Thorlabs PDA100A), a Lock-in amplifier (SR830) and a multimeter (Keithley 2000) was used.

UPS and XPS spectra were collected by a Thermo Scientific ESCALAB 250Xi with an ultra-high vacuum below than $10^{-10}$ torr. A He I $(21.22 \mathrm{eV})$ and an $\mathrm{Al} \mathrm{k} \alpha$ radiation source $(1,466 \mathrm{eV})$ with an experimental resolution of $0.1 \mathrm{eV}$ were used for the UPS measurement and the XPS measurement, respectively.

AFM measurements of PEDOT:PSS films were conducted on a Cypher S Atomic Force Microscope.

Raman spectra were collected by Renishaw inVia-Reflex.

The thicknesses of the films were characterized using a KLA Tencor P-7 Stylus Profiler. 


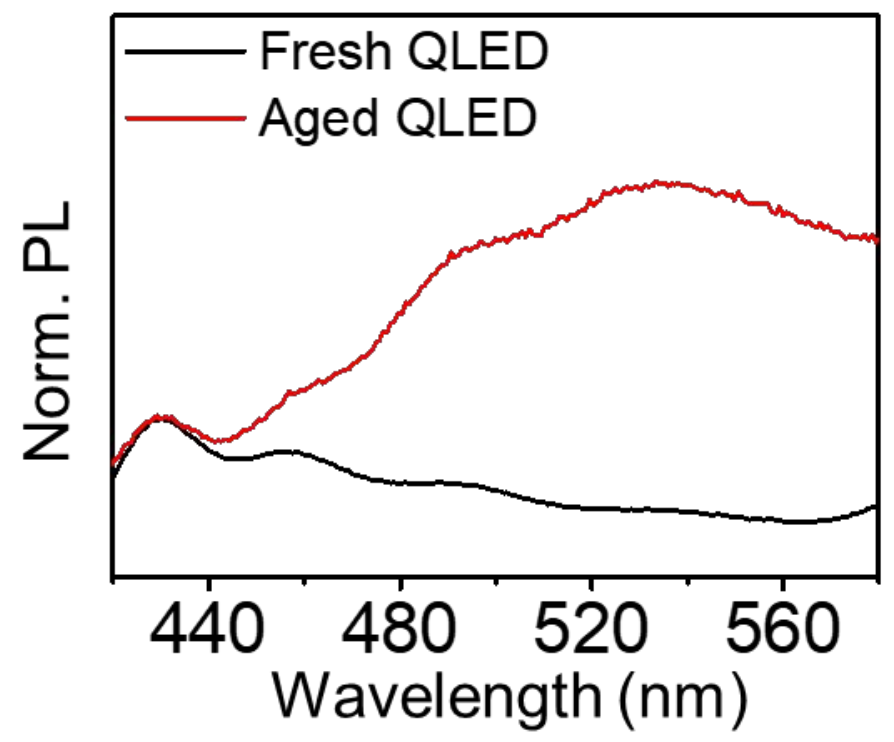

Figure S1. PL spectrum of a QLED before electrical aging and after aging at $50.0 \mathrm{~mA} \mathrm{~cm}-2$ for 100 hours. 


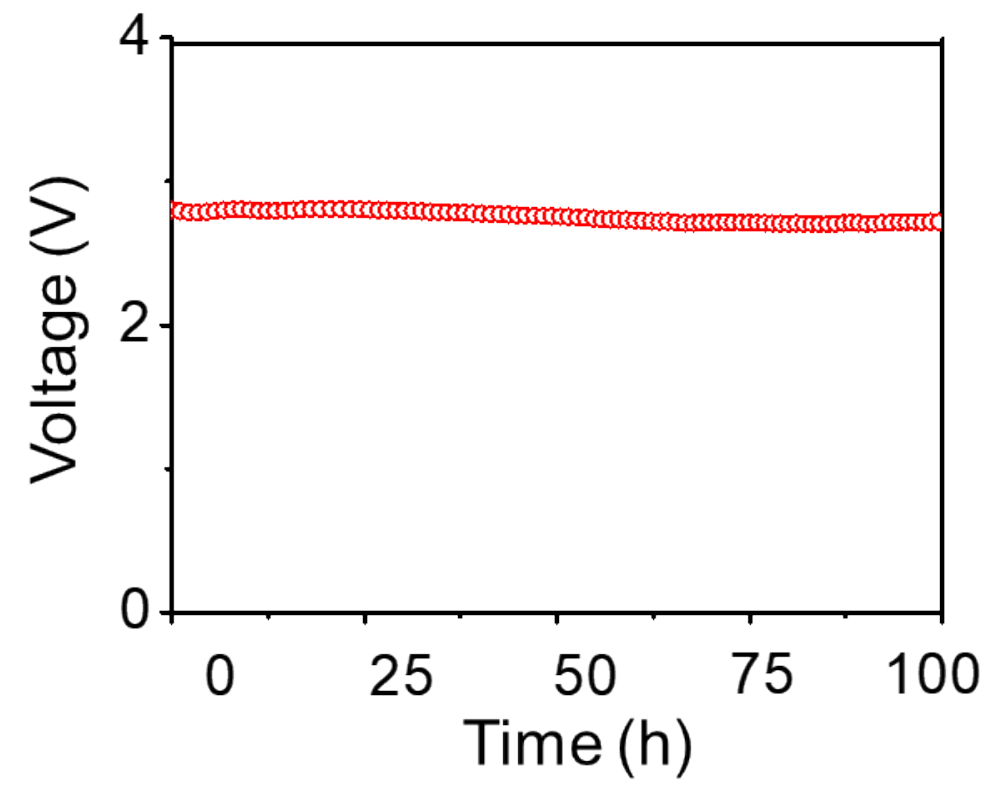

Figure S2. The driving voltage of a hole-only device aging at $50.0 \mathrm{~mA} \mathrm{~cm} \mathrm{c}^{-2}$ for 100 hours. 


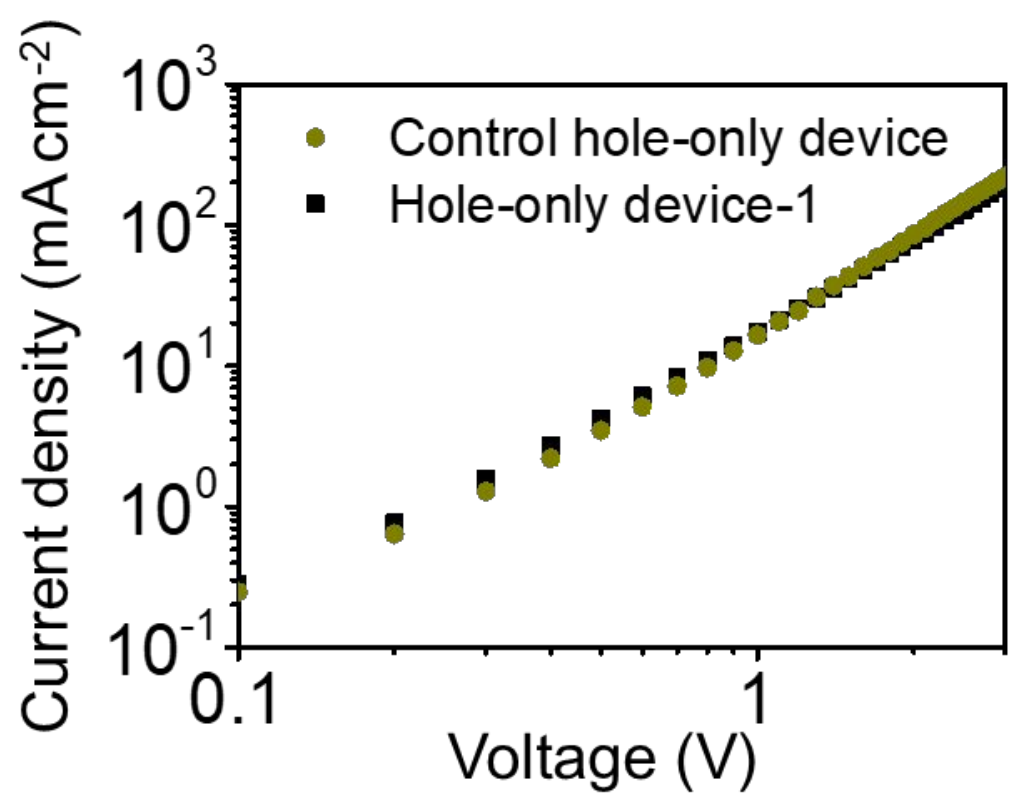

Figure S3. J-V characteristics for the hole-only devices with a structure of ITO/PEDOT:PSS/TFB/MoO $/ \mathrm{Au}$. The control hole-only device was fabricated directly by depositing the PEDOT:PSS layer, the TFB layer and the $\mathrm{MoO}_{\mathrm{x}} / \mathrm{Au}$ electrode, without the transformation processes from the bi-polar device to the hole-only device. Hole-only devices-1 was fabricated by the transformation from the bi-polar device to the hole-only device. 
a

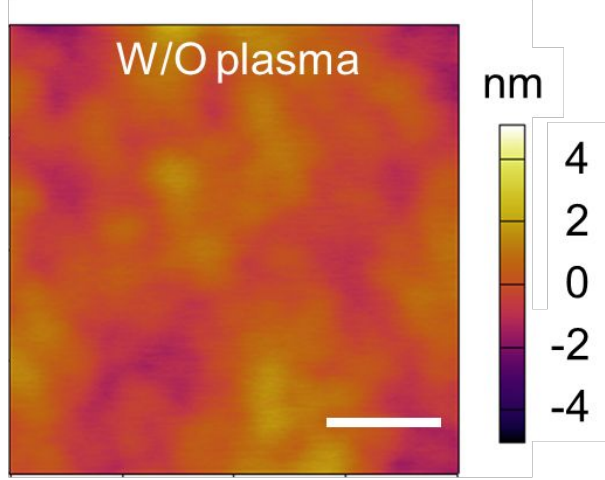

b

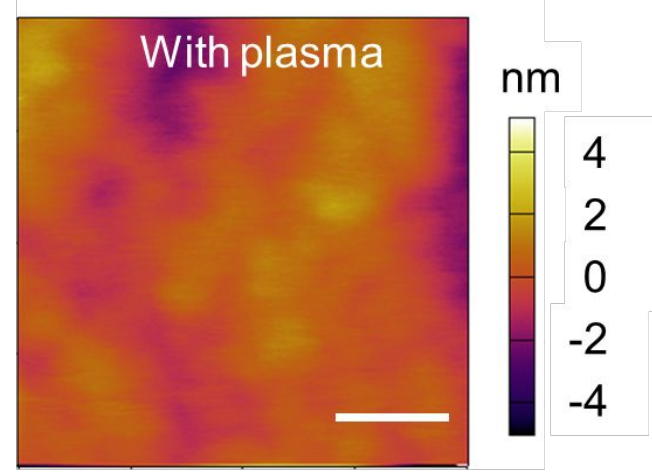

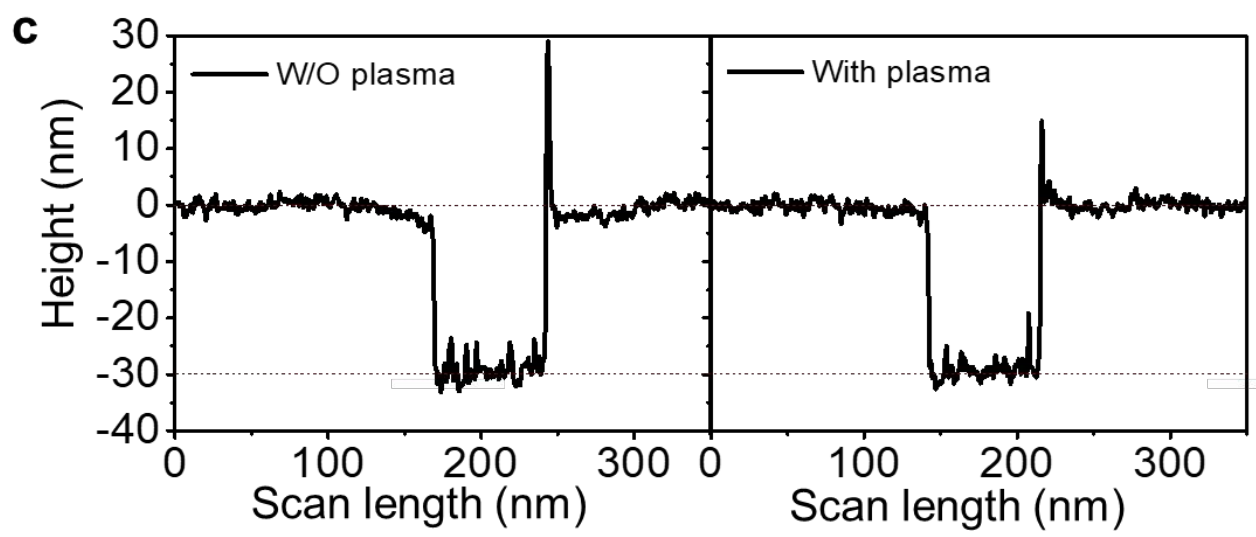

Figure S4. Atomic force microscopy images for (a) the pristine PEDOT:PSS film and (b) the plasma-treated PEDOT:PSS film. Scale bar: $50 \mathrm{~nm}$. (c) Thicknesses of the pristine and the plasma-treated PEDOT:PSS measured by Stylus Profiler. 


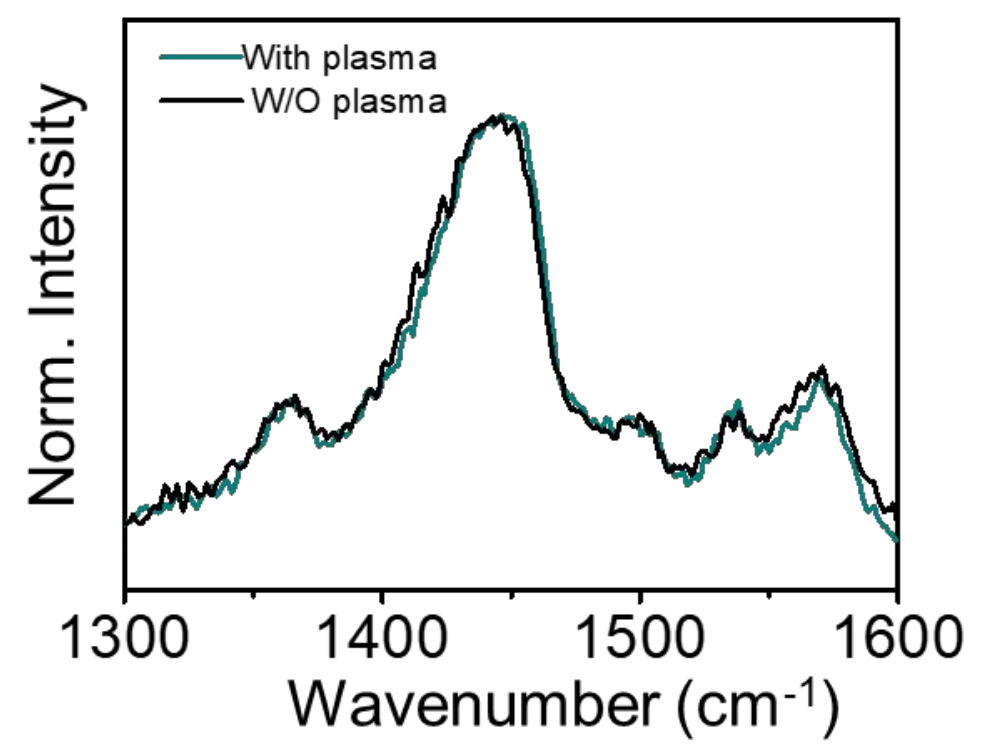

Figure S5. The Raman spectrum of a pristine PEDOT:PSS film and a plasma-treated PEDOT:PSS film. 


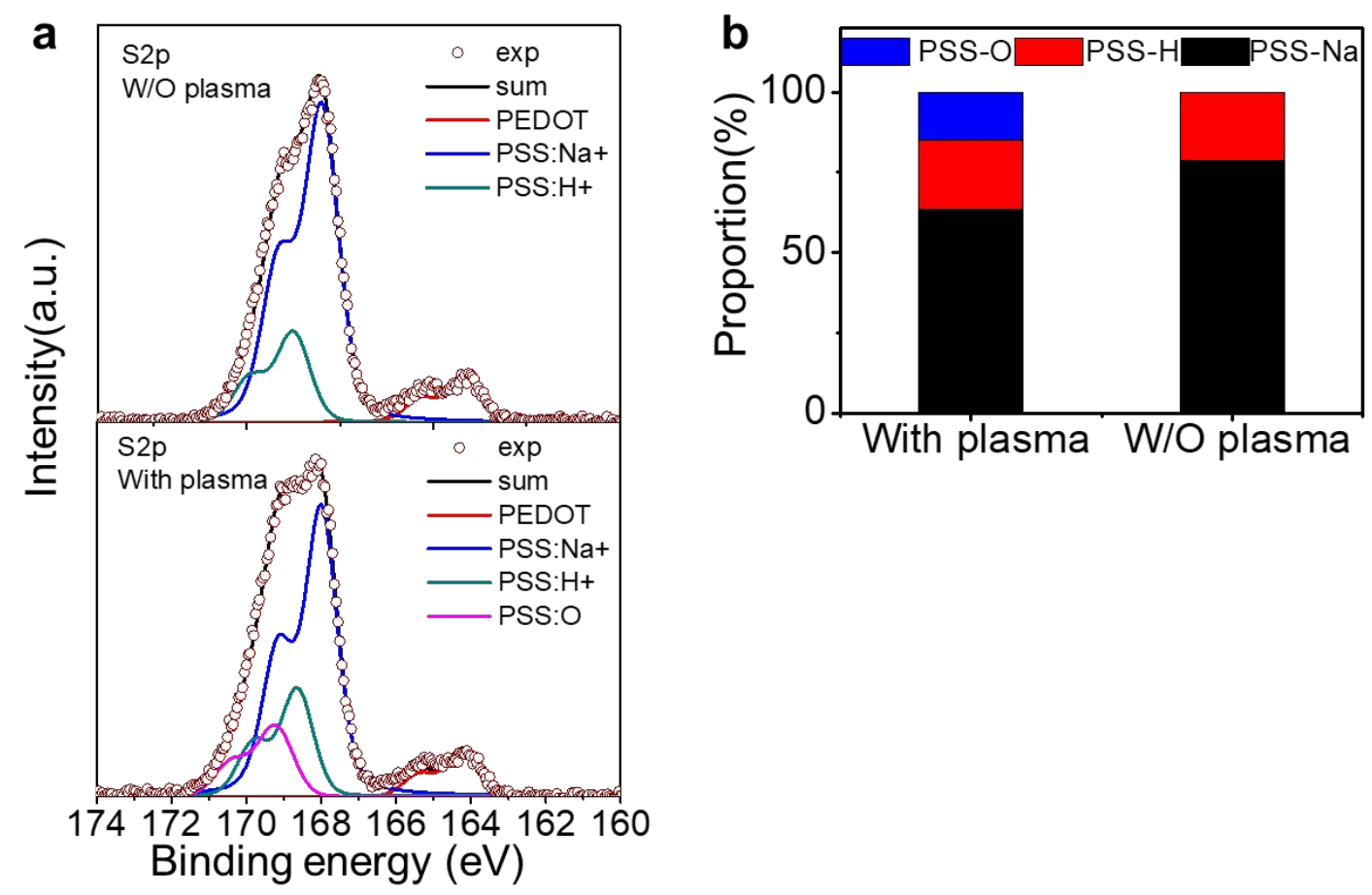

Figure S6. (a) S $2 p$ core spectra of the pristine and the plasma-treated PEDOT:PSS film. Open circles and black lines show measured spectra and sum-fitted curves, respectively. Red lines, blue lines, cyan lines and pink lines are fitted curves for each bond: PEDOT, PSS-Na+, PSS-H+, and PSS-O. (b) The proportion of PSS-O, PSS-H and PSS-Na for the pristine and the plasma-treated PEDOT:PSS. 


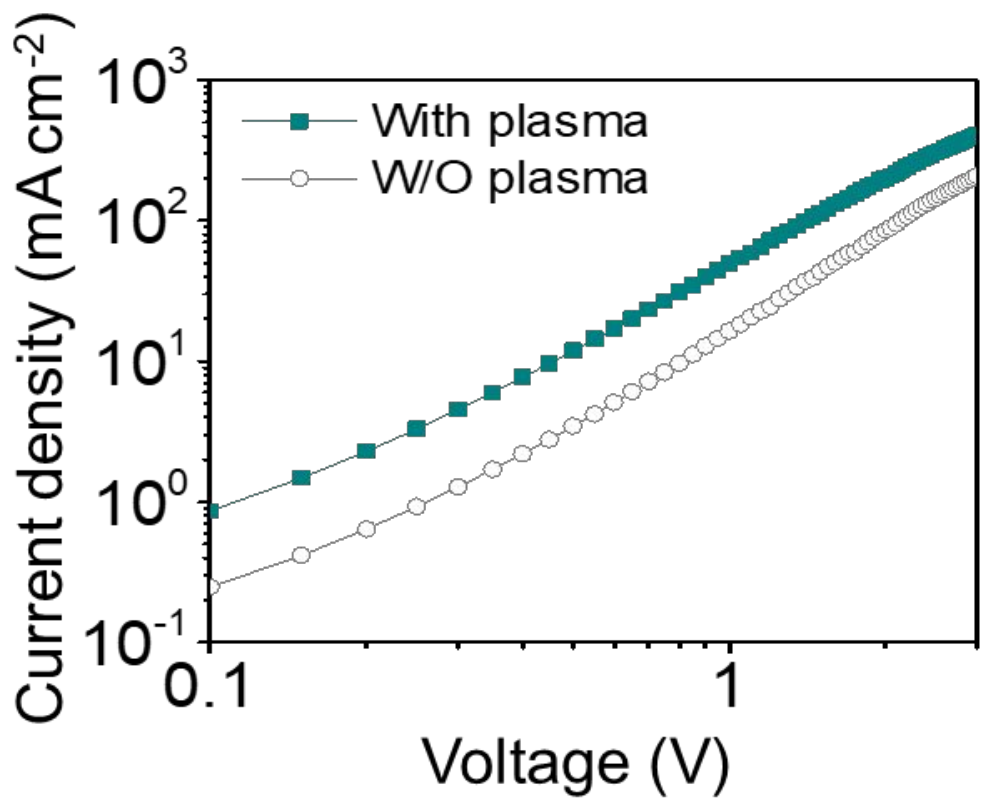

Figure S7. J-V characteristics of the hole-only devices (ITO/PEDOT:PSS/TFB/MoOx/Au) with the pristine PEDOT:PSS HIL or the plasma-treated PEDOT:PSS HIL. 


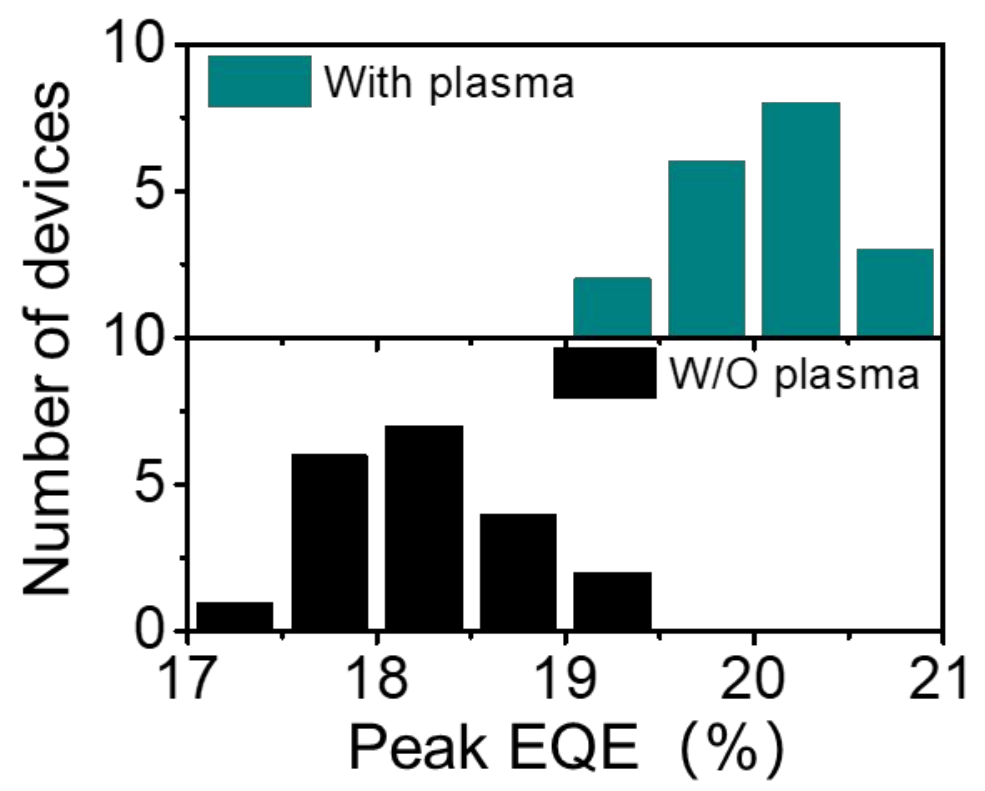

Figure S8. Histogram of peak EQEs for the red QLEDs with the pristine PEDOT:PSS HIL or the plasma-treated PEDOT:PSS HIL. 


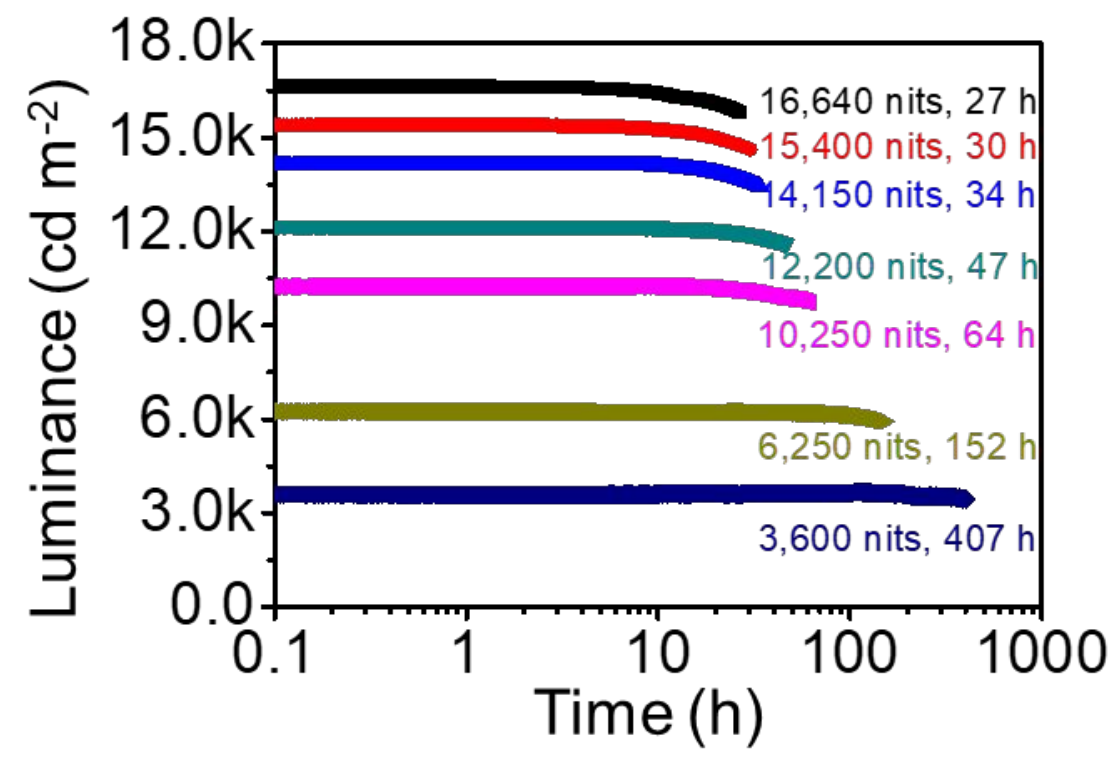

Figure S9. $T_{95}$ operational lifetimes at different initial brightness for the red QLEDs with the plasma-treated PEDOT:PSS HIL. 


\section{REFERENCES}

(1) Spanhel, L.; Arpac, E.; Schmidt, H. Semiconductor Clusters in the Sol-gel Process: Synthesis and Properties of CdS Nanocomposites. J. Non-Cryst. Solids 1992,147, 657-662.

(2) Meulenkamp, E. A. Synthesis and Growth of ZnO Nanoparticles. J. Phys. Chem. B 1998, 102(29), 5566-5572. 